\title{
Early Predictors of the Endometrium Cancer Recurrence in Our Environment
}

\author{
Lopez Gonzalez EIga, Montero Venegas Francisco Manuel, Camacho Carrasco Maria
}

Juan Ramon Jimenez Hospital, Huelva, Spain

\section{Email address:}

elga_t@hotmail.com (L. G. Elga)

\section{To cite this article:}

Lopez Gonzalez Elga, Montero Venegas Francisco Manuel, Camacho Carrasco Maria. Early Predictors of the Endometrium Cancer Recurrence in Our Environment. Journal of Cancer Treatment and Research. Vol. 9, No. 2, 2021, pp. 22-26. doi: 10.11648/j.jctr.20210902.11

Received: May 26, 2021; Accepted: June 8, 2021; Published: June 16, 2021

\begin{abstract}
Objective: to find out the predictors in the recurrence of the illness in patients suffering from endometrium cancer at the differet stages of it. Material and Method: Retrospective study of 170 patients who were diagnosed with endometrium cancer between 2013-2017 at "Juan Ramon Jimenez" Hospital in Huelva. Variables such us age, degree of tumour differentation, miometrial invasion (MI), post surgical staging and lympho-vascular space infiltration (LVSI) were analysed in all those patients that had recurrences during the period of the study. The study was sone by using SPSS 23rd V. Once the analysis of Kolmorov-Smirnov concluded in an unusual result; the Chi-Squared test was used in order to obtain parametric categorical data; whereas the $\mathrm{U}$ (by Mann Whitney) test was used for the non-parametric data. $\mathrm{P}<0.01$ was accepted as significant from a statistic point of view. results: During the study, 9 patients were diagnosed with tumoral affects recurrence. This means $5.3 \%$ out of the whole sample. The statistic analysis concluded that there is no relation of dependence among the recurrence varible and the Miometrial Invasion variable $(\chi 2=4.780, p=0.092)$ recurrence and Tumor grade $(\chi 2=7.765, p=0.051)$ and recurrence and post surgical $(\chi 2=10.200, p=0.070)$. On the contrary, it is stated that there is a relation of dependence among lymph-node damage variable and LSVI $+(\chi 2=9.954, \mathrm{Cc}=0.235, \mathrm{p}<.01)$. The existence of LSVI was evaluated in all the patients. IVSI was negative in 141 cases and 4 of these cases had recurrence of the illness $55.5 \%$ of the patients in which the illness recurred had positive LVSI (5 out of 9). Conclusions: our results proved that more than a half of the patients with recurrence of the illness have Lympho-vascular infiltration and also; if the LVSI is negative, there is a risk of $2.8 \%$ of having recurrence. Although it would be neccessary to carry out prospective multi-center studies, this preliminary study determines an obvious relation between LVSI and the recurrence in a short or long term period. Again, The variable that stablishes the LVSI can be a relevant datum when dealing with the staging process of the endometrium cancer, apart from being very useful in the prediction of the illness recurrence.
\end{abstract}

Keywords: Cancer, Endometrium, Laparoscopy, Lymphovascular Infiltration, Lymphadenectomy, Survival

\section{Introduction}

With an incidence of $7 \%$, endometrial cancer is the most frequent gynecological neoplasm in developed countries, it also has a mortality rate of $4 \%$, and has an average age of presentation of 62 years and death at 71 year [1].

Regarding adenocarcinomas, we can confirm, as described in the bibliography and what was observed in our patients, that up to $75 \%$ occur during the postmenopausal period, becoming especially relevant during the sixth and seventh decade of life. In those patients in whom it occurs in the premenopausal stage, we observe that there are $5 \%$ who are under 40 years of age at the time of diagnosis [2].
The staging of the disease is given by surgery, as confirmed by the guidelines.

One of the main causes involved in carcinogenesis is exposure to estrogens, since most of the risk factors are related to both endogenous and exogenous exposure of the endometrium to estrogens: thus, hormone replacement therapy, anovulation cycles and estrogen-secreting tumors are fundamental causes implicated in the disease [3].

On the contrary, it has been observed that factors that decrease estrogen exposure tend to protect against disease, such as smoking and oral contraceptives.

In addition, other risk factors such as obesity, nulliparity, late menopause, diabetes mellitus, high blood pressure, 
family history, diet rich in animal fats and history of pelvic irradiation must be taken into account, since they are crucial for the development of the disease [4].

By now, according to the NCCN guidelines [5], the accepted treatment for endometrial cancer regardless of the stage in which we find ourselves is the performance of total hysterectomy with bilateral salpingo-oophorectomy and pelvic and para-aortic lymphadenectomy.

In numerous studies, pelvic and para-aortic lymph node involvement has been found in the final results of pathological anatomy in patients who initially, in the preoperative study, were considered as stages I and II [6]. In addition, a para-aortic involvement of the 50 to $72 \%$ in those patients with pelvic lymph node infection.

Even so, there is controversy about performing para-aortic lymphadenectomy in low-risk patients, these being defined as those with myometrial invasion $<50 \%$, absence of lymphovascular invasion (ILV), endometrial histology of grades 1 and 2 and tumor size smaller than $2 \mathrm{~cm} \mathrm{[7].}$

On the other hand, taking into account that the recurrence rate of early stage endometrial cancer ranges between 3 and $17 \%$, the influence of primary and adjuvant treatment and that most recurrences $(75-80 \%)$ occur outside the pelvis, and most occur two to three years after treatment, $64 \%$ after two years and $87 \%$ after three years, we think it is important to carry it out, in order to try to reduce the recurrence rate by minimum possible.

Hence the need to carry out this study arises, which has the purpose of evaluating our results, combining the characteristics of the population attended and knowing what are the factors that can help us predict the recurrence of the disease in our patients, putting in value lymphovascular infiltration, even in the earliest stages. In this way, we will be able to carry out protocols that help us to intuit the evolution and prognosis of the disease in our population.

\section{Material and Method}

Retrospective study of 170 patients diagnosed with endometrial cancer diagnosed in the 2013-2017 period at the Juan Ramón Jiménez Hospital in Huelva. Variables such as age, degree of tumor differentiation, myometrial invasion, postsurgical stage and infiltration of the lymphovascular space (LVSI) were analyzed; the study population was those patients who presented recurrence in the period studied, in $80 \%$ of them during 5 years. All those patients in whom surgical treatment was not performed and those in which the status of the lymphovascular space was not reported were excluded.

The patients underwent appropriate surgery according to the FIGO stage at diagnosis, performing second-stage surgery in those who required re-staging after surgical treatment. Hysterectomy with bilateral salpinguectomy and abdominal cavity evaluation was performed in all cases, with omentectomy in patients with type II endometrial cancer and lymphadenectomy in intermediate and high risk cases and in all type II cases. Low-risk patients did not receive any adjuvant treatment but did receive follow-up for the next 5 years after surgery, six-monthly and annually consecutively.

For the analysis of the lymphovascular space, it was considered positive in all those patients with the presence of tumor cells in the endothelial space outside the immediate invasive border, evaluated in all cases by pathologists with years of experience.

Using recommended guidelines, the pathologists reported the following: types of histopathology; tumor grade; invasion of the myometrium (no invasion, more or less than 50\%); involvement of the cervix (glandular / stromal invasion), parametrium, vagina, ovaries, tubes, omentum; and number of pelvic and para-aortic lymph nodes, including number of metastatic nodes. The stage of the disease was reported according to the FIGO classification. All registered recurrences were histopathologically verified.

Statistical analysis was performed with the SPSS 23v program. Having performed a Kolmogorov-Smirnov analysis and after obtaining a non-normal result, the chi-square test was used for categorical parametric data, and the independent Mann-Whitney U test was used for non-parametric data. A p $<0.01$ was accepted as statistically significant.

\section{Results}

During our study period, a total of 170 cases of endometrial cancer were detected between January 2017 and December 2020.

The average age was 64 years with a median of 58 years. $14 \%$ were 50 years old or younger and only $1.8 \%$ were over 80 years old, with the highest percentage of the disease being concentrated in the age group between 61 and 70 years (Table 1).

Table 1. Age of presentation.

\begin{tabular}{ll}
\hline Age & $\mathbf{N}(\%)$ \\
\hline$<40$ years & $6(3.5)$ \\
$41-50$ years & $18(10.5)$ \\
$51-60$ years & $53(31.2)$ \\
$61-70$ years & $71(41.8)$ \\
$71-80$ years & $19(11.2)$ \\
$\geq 81$ años & $3(1.8)$ \\
Total & $170(100)$ \\
\hline
\end{tabular}

In $86.5 \%$ of the patients, metrorrhagia stands out as the main reason for consultation. (Table 2).

In turn, we detected that up to $50.6 \%$ obtained diagnostic confirmation by hysteroscopy and $44.7 \%$ by endometrial aspirate performed in consultation.

The most frequent histology found has been endometrioid carcinoma with $84.1 \%$ (Figure 1) of the cases and of these, up to $17.1 \%$ infiltration of the lymphovascular space has been detected. 
Table 2. Reason for Consultation.

\begin{tabular}{lllll}
\hline & Frequency & Percentage & Valid percentage & Accumulated percentage \\
\hline Metrorrhagia & 147 & 86.5 & 86.5 & 86.5 \\
Abdominal pain & 7 & 4.1 & 4.1 & 90.6 \\
Casual Finding & 15 & 8.8 & 8.8 & 99.4 \\
Tamoxifen & 1 & 0.6 & 0.6 & 100.0 \\
Total & 170 & 100.0 & 100.0 & \\
\hline
\end{tabular}

Considering the stage of the disease at the time of diagnosis, $54.1 \%$ were detected in stage IA followed by stage IB with $22.4 \%$ of the cases and according to this, we observe that the most frequently performed treatment is Hysterectomy + double adnexectomy in the $61.2 \%$ of cases, followed by it together with pelvic lymphadenectomy (Table 3 ).

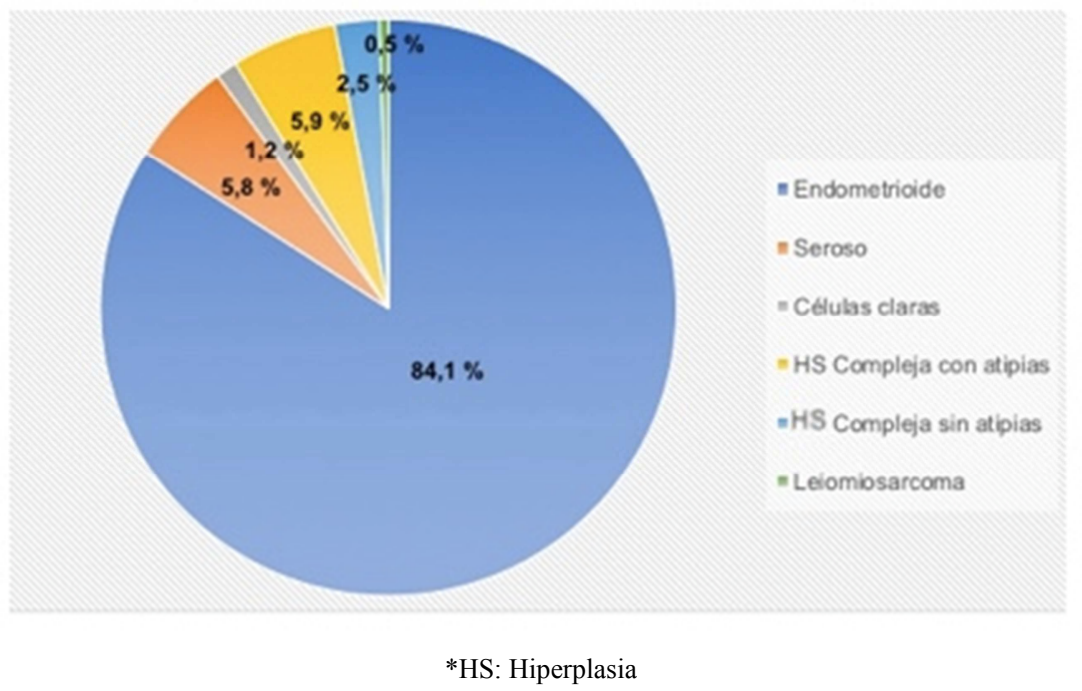

Figure 1. Histological type.

Table 3. Treatment performed.

\begin{tabular}{lllll}
\hline & Frequency & Percentage & Valid percentage & Accumulated percentage \\
\hline No treatment & 10 & 5.9 & 5.9 & 61.2 \\
HT + DA & 104 & 61.2 & 12.4 & 67.1 \\
HT + DA + LP & 21 & 12.4 & 20.6 & 79.4 \\
HT + DA + LPAo & 35 & 20.6 & 100.0 \\
Total & 170 & 100.0 & 100.0 \\
\hline
\end{tabular}

HT + DA: Total Hysterectomy + Double Adnexectomy

HT + DA + LP: Total Hysterectomy + Double Adnexectomy + Pelvic lymphadenectomy

HT + DA + LPAo: Total Hysterectomy + Double Adnexectomy + Para-aortic lymphadenectomy

On the other hand, we have detected that $53.5 \%$ of the cases presented a myometrial invasion greater than $50 \%$ and that $55.9 \%$ of the cases were in stage G1.

The most used approach has been laparoscopic, being performed in $91.2 \%$ of cases, having to perform a laparotomy only in $3 \%$ of cases. The remaining $5.8 \%$ did not undergo surgery due to comorbidities and anesthetic risk of the patients. Lymphadenectomy was performed in $32.9 \%$ of the cases, finding metastases at the pelvic level in $2.4 \%$ and at the para-aortic level in $3.5 \%$ of the patients.

Of the 170 women diagnosed, 5 patients $(2.94 \%)$ have died during the follow-up period, 156 (91.76\%) remain disease-free at the time of the study, and 9 patients $(5.3 \%)$ have relapsed.

Of these patients in whom the disease has recurred, we found that 8 patients had a myometrial invasion $>50 \%$ and only 1 patient had a myometrial involvement $<50 \%$.
Furthermore, considering the tumor grade, we have observed 2 cases in G1, 3 cases in G2 and 4 cases in G3. As for the stage of the disease, we have detected 3 cases in stage IV, 2 cases in stage III, 2 cases in stage IIA and 2 cases in stage IB.

Taking into account these results, the statistical analysis has revealed that there is no dependency relationship between the variables Recurrence and Myometrial Invasion $(\chi 2=4.780$, $\mathrm{p}=.092)$, recurrence and Tumor Grade $(\chi 2=7.765, \mathrm{p}=.051)$ and recurrence and the postsurgical stage $(\chi 2=10.200$, $\mathrm{p}=.070)$.

On the contrary, it is observed that there is a dependency relationship between the variables Lymph node involvement and LSVI $+(\chi 2=9.954, \mathrm{Cc}=.235, \mathrm{p}<.01)$.

In all the patients studied, including those at low risk, the existence of lymphovascular infiltration was evaluated. This was negative in 141 cases and despite this, we found 4 cases 
with recurrence of the disease. However, we can affirm that $55.5 \%$ of the patients with disease recurrence had positive LVSI ( 5 of 9 patients).

\section{Discussion}

Endometrial cancer is one of the most prevalent cancers in our population, and in our country it is the third major gynecological cancer, after breast and cervical cancer [8].

Our results allow us to put into context a population of 170 patients with endometrial cancer in which it stands out that the age of presentation occurs mainly between 51 and 70 years, with an average of 64 years, debuting in a high percentage of the population in Stage I. These findings are consistent with the international literature that shows that this neoplasm presents good clinical results, especially when it presents in low-risk stage [9].

According to our results, the incidence of endometrial cancer is rare before 50 years $(14 \%)$ and very low over 80 years $(1.8 \%)$. However, it is noteworthy that $14 \%$ of the cases affected premenopausal women. The occurrence of cancer in this age group could partly explain the increase in its incidence in the country and the world. The risk factors that could explain the incidence in this age group would be; The decrease in parity, anovulatory cycles and morbid obesity. In addition, possible hereditary and genetic defects should also be considered in early-onset cancers, highlighting Lynch syndrome, microsatellite instability and specific mutations that mainly affect P53, B-catenin, P-ten and K-ras.,among others [10].

The most frequent symptom observed in women with this type of cancer is metrorrhagia, which, in women who still have menstrual cycles, is usually intermenstrual and less frequently appears as an alteration in the amount or duration of menstruation. Thanks to this characteristic, endometrial cancer is diagnosed in earlier stages and has a more favorable course than ovarian and cervical cancer. This symptom has been the main one, with $86.5 \%$ of the cases, a figure very similar to that reported in other series $(80 \%)$. Something different happens with abdominal pain (4\% in our population versus $22 \%$ ) [11]. The difference may be in the group of women who present the combination of both symptoms and that we have analyzed independently.

Regarding diagnosis, it should be noted that ultrasound has had great limitations as a diagnostic method for endometrial adenocarcinoma.

The main diagnostic method was the Gineaspir aspiration cannula biopsy and, secondly, hysteroscopy. A meta-analysis has reported that the cannula was the best device, since it presented detection rates for endometrial cancer in postmenopausal and premenopausal women of $99.6 \%$ and $91 \%$ respectively, which is why it is currently considered the method of choice for the performance of an initial biopsy [12].

If we look at the national database of the United States 2012 , it is observed that $69.6 \%$ of endometrial cancers are diagnosed in stage I, $7.1 \%$ in stage II, $8.3 \%$ in stage III and $6.7 \%$ in stage IV [13]. In our casuistry, the proportion of patients in stage I is slightly higher $(76.6 \%)$, with a lower proportion in the rest of the stages.

The endometrioid variety was the most frequent histological type $(84.1 \%)$. However, the serous papillary, clear cell and undifferentiated varieties were presented at relatively low frequencies, consistent with what was observed in the literature [14].

Lymphadenectomy was performed in $39.2 \%$ of the cases, finding involvement at the pelvic level in $2.4 \%$ of the cases and involvement at the aortic level in $3.5 \%$ of the cases.

The recurrence rate described in the medical literature is low (0-29\%), [15] but the follow-up in these studies is for a short period of time. In our case, the recurrence rate was $5.3 \%$, but possibly if we increase the follow-up, this rate would be higher, as in other reviews.

In addition, we have not shown that there is a dependency relationship between the variables of recurrence and myometrial invasion and recurrence and the postsurgical stage, although we have observed it between the variables of lymph node involvement and LSVI + , a fact that makes us highlight the importance of carrying out a study of lymph node involvement and lymphovascular infiltration, even in incipient stages of the disease, in order to minimize and determine which patients are at higher risk of developing a recurrence of the disease.

\section{Conclusion}

Endometrial cancer is the most common gynecological cancer in women, its most common form of presentation being metrorrhagia and its main diagnostic method is endometrial aspirate or, failing that, hysteroscopy.

The most frequent type is endometrioid carcinoma, being detected in early stages and showing almost $20 \%$ lymphovascular infiltration, even in these early-stage patients in whom no adjuvant treatment has been performed.

our results showed that more than half of the patients with disease recurrence have lymphovascular infiltration and furthermore, if the LVSI is negative, we have a $2.8 \%$ risk of suffering a recurrence. Although it would be necessary to carry out multicenter prospective studies, this preliminary study determines a clear relationship between LVSI + and recurrence in the short and long term. Again, the variable that establishes the LVSI + may be a relevant piece of information for the staging of endometrial cancer, in addition to showing utility in predicting disease recurrence.

Likewise, we are aware of the limitations of a retrospective study, so we are working to develop more studies that confirm these conclusions and can reaffirm the data presented.

\section{References}

[1] Ferlay J, Soerjomataram I, Dikshit R, Eser S, Mathers C, Rebelo M, Parkin DM, Forman D, Bray F. Cancer incidence and mortality worldwide: sources, methods and major patterns in GLOBOCAN 2012. Int J Cancer. 2015 Mar 1; 136 (5): E359-86. https://doi.org/10.1002/ijc.29210. 
[2] Endometrial cancer. En: Callahan TL, Caughey AB, editores. Blueprint's obstetrics and gynecology, 5th ed. Philadelphia, PA: Lippincott Williams \& Wilkins; 2008. p. 310-6.

[3] Ramondetta LM, Burke TW, Broaddus R. Treatment of endometrial cancer. In: Aman UB, Ralph SF (editors). Gynecologic Cancer. M. D. Anderson. Cancer Care Series. USA: Springer; 2006. p. 148-174.

[4] Zaino RJ. Introduction to Endometrial Cancer. In: Giordano A, Bovicelli A, Kurman RJ (editors). Molecular Pathology of Gynecologic Cancer. USA: Humana Press; 2007. p. 51-100.

[5] Zhang H, Zuo Z, Wang Y, Wang L, Cohort study evaluating paraaortic lymphadenectomy in endometrial cancer. Oncol Lett. 2012; 4 (6): 1361-5.

[6] Convery PA, Cantrell LA, Di Santo N, Broadwater G, et al. Retrospective review of an intraoperative algorithm to predict lymph node metastasis in low-grade endometrial adenocarcinoma. Gynecol Oncol. 2011; 123 (1): 65-70.

[7] Alay I, Turan T, Ureyen I, Karalok A, et al. Lymphadenectomy should be performed up to the renal vein in patients with intermediate-high risk endometrial cancer. Pathol Oncol Res. 2015; 21 (3): 803-10.

[8] Hahn HS, Lee IH, Kim TJ, Lee KH, et al. Lymphovascular space invasion is highly associated with lymph node metastasis and recurrence in endometrial cancer. Aust N Z J Obstet Gynaecol. 2013; 53 (3): 293-7.

[9] Pecorelli S. Revised FIGO staging for carcinoma of the vulva, cervix, and endometrium. Int J Gynaecol Obstet. 2009; 105 (2): 103-4. https://doi.org/10.1016/j.ijgo.2009.02.012.
[10] Balagueró L, Comino R, Sainz de la Cuesta R, Jurado M, Xercavins J, Petschen I. Carcinoma de endometrio. Documento de Consenso SEGO. Madrid: Sociedad Española de Ginecología y Obstetricia; 1999. p. 91-136. https://doi.org/10.1016/j.gine.2009.03.009.

[11] Hecht JL, Mutter GL. Molecular and pathologic aspects of endometrial carcinogenesis. J Clin Oncol 2006; 10 (29): 478391. https://doi.org/10.1200/JCO.2006.06.7173.

[12] Sanhueza P, Oliva L. Eficiencia de los métodos diagnósticos en el estudio del sangrado uterino anormal en la peri y post menopausia. Rev Chil Obstet Ginecol 2008; 73 (1): 58-62. https://dx.doi.org/10.4067/S0717-75262008000100010.

[13] Tabor A, Watt HC, Wald NJ. Endometrial thickness as a test for endometrial cancer in women with postmenopausal vaginal bleeding. Obstet Gynecol 2002; 99 (4): 663-70. https://doi.org/10.1016/S0029-7844(01)01771-9.

[14] J. A. Lachance, E. N. Everett, B. Greer, L. Mandel, E. Swisher, H. Tamini, et al. The effect of age on clinical/pathologic features, surgical morbidity, and outcome in patients with endometrial cancer. Gynecol Oncol, 101 (2006), pp. 470-475. https://doi.org/10.1016/j.ygyno.2005.11.009.

[15] Siegel R, DeSantis C, Virgo K, Stein K, Mariotto A, Smith T, et al. Cancer treatment and survivorship statistics, 2012. CA $\begin{array}{lllll}\text { Cancer J Clin 2012; } 62 & \text { (4): 220-41. }\end{array}$ https://doi.org/10.3322/caac.21149. 